Kolonialverwaltung der frühen 1900er-Jahre zurückverfolgen (Copnall, 2017: 226). Er entspringt dem Versuch, die die Kolonialmacht herausfordernden eingesessenen und zutiefst strategischen Verhaltensweisen der kolonialen Subjekte zu verstehen, $\mathrm{zu}$ benennen und zu typologisieren.

Tatsächlich hat Tajility zwei Dimensionen, eine strukturelle und eine individuelle. Warum handeln und entscheiden, wenn es zu einem späteren Zeitpunkt bessere Optionen geben mag? Das Nichthandeln kann aber ebenso gut mit eingeübten Verhaltensweisen im Zusammenhang stehen. Warum handeln und entscheiden, wenn es nicht behagt? Die strategische Komponente hat Alex de Waal (2008) als Politik der Erschöpfung beschrieben. Ein solches Auswarten kann jemanden in eine unerwartet vorteilhafte Position spülen. "Delay is always an option, and those who use the skill of tajility [...] most adroitly are likely to be the ones with most energy left at the end of the day. Nothing is guaranteed until it happens." Diesen letzten Satz nicht als Bedrohung, sondern als immerwährende Chance zu begreifen, ist der Kunst des südsudanesischen Wartens immanent. Wer sagt schließlich, dass es zu einem späteren Zeitpunkt nicht besser wird? Letzten Endes ist Warten praktisch umgesetzter Optimismus. Warten ist eine stetige Wette auf die Zukunft.

\title{
Footing
}

Unzweifelhaft ist das spezifisch südsudanesische Verhältnis zu Zeit von den räumlichen Gegebenheiten geprägt. Wie kann Zeit verlässlich vereinbart werden, wenn Autos nach einem Regen selbst in Jubas Botschaftsviertel Tongping stecken bleiben? Mitunter für Tage. Regen- und Trockenzeiten waren immer der bestimmende Rhythmus im Südsudan. Denn in der Regenzeit wird selbst die einzige verlässliche Fortbewegungsform in weiten Teilen des Landes schwierig: das Gehen, oder in südsudanesischem Englisch: »Footing«.

Gehen und warten - »Footing« und »Waiting« - waren und sind auch die zwei dominierenden Modi der Kriegsführung im Südsudan, die nur kurz durch Momente der Turbulenz unterbrochen werden. "Ich habe das ganze Land zu Fuß kennengelernt«, erzählt Kuot von seiner Zeit bei der SPLA. »Wir sind von Equatoria nach Wau marschiert. Dann kam ein neuer Befehl, und wir sind hinüber nach Upper Nile. Und dann nach Äthiopien. Und dann bis hinunter nach Uganda. Alles zu Fuß.« Der scheinbare Nachteil der Geschwindigkeit verkehrt sich in den herausfordernden Bedingungen eines tropischen, infrastrukturell nicht erschlossenen Landes bald in Vorteile wie Verlässlich- 
keit und Flexibilität. Gegen geübte Marschierer:innen kommt selbst eine geölte und hochgerüstete militärische Maschinerie schwer an. Zugleich sind Anstrengung und Dauer die unerbittlichen Begleiter von Footing and Waiting, eine beständige Quelle von Frustration. »[O]ne ex-combatant described fighting in Unity state and then walking some $600 \mathrm{~km}$ to Nasir, near Ethiopia, before defecting.« (Boswell, 2019: 9)

Doch abgesehen von der Alternativlosigkeit sind die Vorteile nicht $\mathrm{zu}$ unterschätzen. Dies mussten schon einige internationale Militäroperationen schmerzlich akzeptieren. So scheiterte die in den Jahren 2010 bis 2012 medial inszenierte Jagd auf die ugandische Lord's Resistance Army (LRA) und ihren Anführer Joseph Kony an deren trivialen Verschwinden im Busch. Die militärisch nicht mehr schlagkräftige Gruppe bewegte sich so flexibel im Grenzgebiet zwischen Uganda, dem Südsudan und der Zentralafrikanischen Republik, selbstverständlich per Footing, dass selbst technologisch hochgerüstete Gegner an ihrer Ausspähung scheiterten.

Auch die Flucht Riek Machars mit seinen Getreuen nach dem Juba Incident im Juli 2016 in die Demokratische Republik Kongo durch mehrere hundert Kilometer Dschungel konnten angesichts der Notwendigkeit der Vermeidung von Straßen nur marschierend erfolgen. Und sie verlief selbst in einer Situation relativer militärischer Unterlegenheit insofern erfolgreich, als sich die SPLM/A-IO-Teile reorganisieren konnten. Ein Teil wurde später nach Khartum ausgeflogen, der größere Teil nahm den nächsten Fußmarsch Richtung Greater Upper Nile in Angriff.

Diese Fokussierung auf die militärstrategische und mitunter sogar taktische Dimension des Footing darf nicht vergessen lassen, dass diese Fortbewegungsart für den Großteil der Menschen im Südsudan alternativlos ist. So gibt es, bis auf wenige Glückliche, die auf Motorräder zurückgreifen können, schlichtweg kein anderes Fortbewegungsmittel. Doch selbst Motorräder und Autos sind in den allermeisten Gegenden außerhalb der Städte in Ermangelung der notwendigen Straßen und Wege oftmals nutzlos, gerade in der Regenzeit. Weites Gehen ist hingegen schon allein aufgrund der großen Zahl an halbnomadisch lebenden Gemeinschaften gängig. Die ausgeprägten regionalen Handelsnetzwerke, etwa zwischen Jonglei, Eastern Equatoria und Gambela in Äthiopien (Thomas, 2015: 255), haben dazu ein Übriges beigetragen. "Footing Trade« ist die im wahrsten Sinne des Wortes einzig gangbare Option. Denn im Gegensatz zur landläufigen Annahme, dass ein Mangel an Infrastruktur und herausfordernde Umweltbedingungen zur Einschränkung 
des Bewegungsradius führen würden, sind die meisten der im Südsudan lebenden Gemeinschaften hochmobil.

Wasser ist einer der treibenden Faktoren vieler Wanderungen. Es muss entweder herangeschafft werden oder das Vieh muss zu Wasserpunkten getrieben werden. In ländlichen Regionen kann der Weg von den Dörfern zu Wasserstellen, der oft Kindern überantwortet ist, da die Erwachsenen mit Feldarbeit gebunden sind, mehrere Stunden dauern. In eine Richtung. Das Gleiche gilt für den Besuch von Märkten oder anderen Orten, wobei neben der langen Dauer der Fußmärsche das mit ihnen verknüpfte Risiko beträchtlich ist. Eine der ungeschriebenen Grundregeln im Südsudan ist daher, kein Footing in der Dunkelheit. Zumindest nicht allein und außerhalb jener eng abgesteckten Territorien, die gut und umfassend einzuschätzen sind. Das gilt auch und gerade in der Hauptstadt Juba, wo selbst Junubin, also Südsudanes:innen, abends für Strecken von wenigen hundert Metern konsequent auf Autos oder Boda Bodas, Motorradtaxis, zurückgreifen.

Besondere Gefahren müssen diejenigen in Kauf nehmen, die sich den Luxus organisierten Transports nicht leisten können, aber zu weiten Wanderungsbewegungen gezwungen sind: Flüchtende und Vertriebene. Genaue Zahlen schwanken, doch haben Krieg und Naturkatastrophen, insbesondere großflächige Überschwemmungen, eine riesige Menge an Menschen in Bewegung gebracht. Mit Ende 2020 berichtet UNHCR von etwa 2 Millionen südsudanesischen Flüchtlingen in umliegenden Ländern, 1,6 Millionen Binnenvertriebenen, sowie über 300.000 Flüchtlingen aus Nachbarländern, die wiederum im Südsudan Zuflucht gefunden haben, vor allem in Unity State und Upper Nile. Die mit diesen Fluchtbewegungen verbundenen Wanderungen sind gefährlich und auszehrend. Gerade in der Regenzeit, in der viele gezwungen sind, drohenden Überschwemmungen auszuweichen, ist es faktisch unmöglich zu schlafen, weil tagelanges Waten durch Wasser kein noch so kurzfristiges Niederlassen zulässt (vgl. etwa die Schilderungen in James, 2007: 134).

Dazu kommt das Risiko von Überfällen, wie es Jok Madut Jok am Beispiel von Rückkehrenden aus dem Nordsudan schildert. »The foot journey takes anywhere between one week to ten days in a rough terrain with no adequate water or food. One could also run into another raiding force coming back from the South, and the fear of being recaptured prevents many people from embarking on this journey.«(Jok, 2010: 71) Auch wenn diese Schilderungen schon länger zurückliegen, grundlegend geändert haben sich die Bedingungen seither nicht. Ebenso wenig geändert hat sich, dass Ausdauer, Durchhalte- und 
Anpassungsfähigkeit oder, wie es neuerdings so schön heißt, Resilienz ein essenzieller Bestandteil südsudanesischen Lebens sind.

\section{Rule by Law}

Südsudan ist kein rechtloses staatliches Gebilde. Auch die Präsidentschaft bedient sich des Rechts in der Ausübung exekutiver Gewalt. Das zentrale Element ist das »Presidential Decree«, jenes Instrument, mit dem der Präsident seine Entscheidungen dem Staatsvolk bekannt gibt und sie zeitgleich in geltendes Recht überführt. »SSBC [South Sudan Broadcasting Corporation] ist langweilig«, meint mein Freund im Council of States. »Ich schalte immer nur zu den Hauptnachrichten um acht Uhr abends ein. Da verkünden sie die Presidential Decrees, falls Salva welche erlassen hat. Das sind die einzigen interessanten Nachrichten, die sie bringen.« Tatsächlich scheint das so zu sein. Jede Personalentscheidung, jede Einrichtung oder Schließung von Institutionen, Gebote und Verbote, selbst die Festlegung der Anzahl der Gliedstaaten erfolgte und erfolgt via von Salva Kiir unterzeichnetem Dekret. Damit wird Recht gesetzt. Oder, wie es ein Präsidentensprecher anlässlich legalistisch vorgebrachter Zweifel vor einigen Jahren formulierte: »This is an administrative order issued by the president, and an administrative order is not subject to approval. ${ }^{1}$

Es gehört zu den Eigenarten des öffentlichen Umgangs mit Formen von Staatlichkeit im Südsudan, dass diese Form der Rechtssetzung weithin schulterzuckend akzeptiert wird. Wie sich dem Präsidenten legal widersetzen, wo er doch das Recht verkörpert? Meine Frage an für gewöhnlich gut informierte Kolleg:innen und Freund:innen nach der rechtlichen Basis dieser Dekrete erntet gleichermaßen Interesse wie Ahnungslosigkeit. »Das ist eine gute Frage«, so lautete die gängige Antwort. In der Tat findet das Presidential Decree als Instrument keine Erwähnung in den Verfassungen des Südsudan und Sudan, weder in der Übergangsverfassung des Südsudan von 2011, der Übergangsverfassung von Sudan und dem südlichen Sudan von 2005, noch in der von Bashir eingeführten sudanesischen Verfassung von 1998. Lediglich im Falle des Versäumnisses des Parlaments, ein gültiges Budget zu verabschieden, or-more-states-violates-peace-deal [14-01-2021]. 